ACT-12/97

CTP-TAMU-33/97

OUTP-97-37P

quant-ph/9708003

\title{
On Quantum Mechanical Aspects of Microtubules
}

\author{
N.E. Mavromatos ${ }^{a}$ and D.V. Nanopoulos ${ }^{b, c, d}$
}

\section{Abstract}

We discuss possible quantum mechanical aspects of MicroTubules (MT), based on recent developments in quantum physics. We focus on potential mechanisms for 'energyloss-free' transport along the microtubules, which could be considered as realizations of Fröhlich's ideas on the rôle of solitons for superconductivity and/or biological matter. In particular, by representing the MT arrangements as cavities, we present a novel scenario on the formation of macroscopic (or mesoscopic) quantum-coherent states, as a result of the (quantum-electromagnetic) interactions of the MT dimers with the surrounding molecules of the ordered water in the interior of the MT cylinders. Such states decohere due to dissipation through the walls of the MT. Transfer of energy without dissipation, due to such coherent modes, could occur only if the decoherence time is larger than the average time scale required for energy transfer across the cells. We present some generic order of magnitude estimates of the decoherence time in a typical model for MT dynamics. Our conclusion is that the quantum coherent states play a rôle in energy transfer if the dissipation through the walls of the MT cavities is fairly suppressed, corresponding to damping time scales $T_{r} \geq 10^{-4}-10^{-5} \mathrm{sec}$, for moderately large MT networks. We suggest specific experiments to test the above-conjectured quantum nature of the microtubular arrangements inside the cell. These experiments are similar in nature to those in atomic physics, used in the detection of the Rabi-Vacuum coupling between coherent cavity modes and atoms. Our conjecture is that a similar Rabi-Vacuum-splitting phenomenon occurs in the absorption (or emission) spectra of the MT dimers, which would constitute a manifestation of the dimer coupling with the coherent modes in the ordered-water environment (dipole quanta), which emerge due to 'super-radiance'.

${ }^{a}$ P.P.A.R.C. Advanced Fellow, Department of Physics (Theoretical Physics), University of Oxford, 1 Keble Road, Oxford OX1 3NP, U.K.

${ }^{b}$ Department of Physics, Texas A \& M University, College Station, TX 77843-4242, USA,

${ }^{c}$ Astroparticle Physics Group, Houston Advanced Research Center (HARC), The Mitchell Campus, Woodlands, TX 77381, USA,

${ }^{d}$ Academy of Athens, Chair of Theoretical Physics, Division of Natural Sciences, 28 Panepistimiou Avenue, Athens 10679, Greece. 


\section{Introduction}

MicroTubules (MT) appear to be one of the most fundamental structures of the interior of living cells [1]. These are paracrystalline cytoskeletal structures which seem to play a fundamental rôle for the cell mitosis. It is also believed that they play an important rôle for the transfer of electric signals and, more general, of energy in the cell.

In this latter respect it should be mentioned that energy transfer across the cells, without dissipation, had been conjectured to occur in biological matter by Fröhlich [2], already some time ago. The phenomenon conjectured by Fröhlich was based on his one-dimensional superconductivity model: in one dimensional electron systems with holes, the formation of solitonic structures due to electron-hole pairing results in the transfer of electric current without dissipation. In a similar manner, Fröhlich conjectured that energy in biological matter could be transfered without dissipation, if appropriate solitonic structures are formed inside the cells. This idea has lead theorists to construct various models for the energy transfer across the cell, based on the formation of kink classical solutions [3].

In the early works no specific microscopic models had been considered [3]. Recently, however, after the identification of the MT as one of the most important structures of the cell, both functionally and structurally, a model for their dynamics has been presented in ref. [4, in which the formation of solitonic structures, and their rôle in energy transfer across the MT, is discussed in terms of classical physics. In ref. [5] we have considered the quantum aspects of this one-dimensional model, and argued on the consistent quantization of the soliton solutions, as well as the fact that such semiclassical solutions may emerge as a result of 'decoherence' due to environmental entanglement, according to recent ideas [6].

The basic assumption of the model used in ref. [5] was that the fundamental structures in the MT (more specifically of the brain MT) are Ising spin chains (one-space-dimensional structures). The interaction of each chain with the neighboring chains and the surrounding water environment had been mimicked by suitable potential terms in the one-dimensional Hamiltonian. The model describing the dynamics of such one-dimensional sub-structures was the ferroelectric distortive spin chain model of ref. [4].

The one-dimensional nature of these fundamental building blocks, then, opens up the way for a mathematical formulation of the chain as a completely intergrable field theory model, characterized by an infinite-number of conservation laws. The latter are associated with global excitation modes, completely delocalised in the chain space-time. This integrability structure proves sufficient in providing a satisfactory solution to memory coding and capacity [5]. Such features might turn out to be important for a model of the brain as a quantum computer.

This formulation allowed the authors of ref. [5] to employ the so-called Liouville (non-critical) string theory approach to decoherence [7], which is a formalism to treat such completely integrable spin systems embedded in a 'two-dimensional quantum gravity environment'. At this point we should stress that such an environment 
does not necessarily represent realistic space-time effects. As stressed in ref. [5], an external stimulus may distort the neighboring space-time of the unpaired spins of the MT dimers, and such a distortion may be effectively described by a two-dimensional 'metric' in the Liouville picture of MT [5], which should not be necessarily identified with a relatistic four dimensional space time metric $\mathrm{t}$.

The coupling of the MT chain with its 'environment' may lead, according to the analysis of ref. [5], to the formation of macroscopic quantum coherent solitonic states. Then, the details of the environment would play a crucial rôle in determining the time scales over which such coherent states could be sustained before collapsing to classical ground states.

Such analyses have yield important results in the past 10, 11, 6, 12, concerning the passage from the quantum to classical world. In particular, in ref. [6] it was suggested that quantum coherent states may appear as a result of decoherence, depending on the nature of the environment [13]. Such states are minimum entropy/uncertainty states, which propagate 'almost classically' in time, in the sense that their shape is retained during evolution. Such 'almost classical' states are termed 'pointer states' by Zurek [6]. In view of the results of ref. [12], concerning the localization of the wave function in open (stochastic) quantum-mechanical systems, the emergence of pointer states, as a result of decoherence, may be interpreted as implying that the localization process of the state vector had stopped at a stage where it is not complete, but is such that the resulting (minimum-entropy) state is least susceptible to the effects of the environment.

Not all environments admit such pointer states [13]. Here lies one of the advantages of viewing the MT system of chains as a completely integrable Liouville-string theory. The model possesses a pointer basis [14, which in ref. [5] has been identified with the quantized (via squeezed-coherent states) soliton solutions of ref. [⿴囗. other advantage lies on the fact that energy is conserved on the average in the model, despite the environmental entanglement. This is for purely stringy reasons pertaining to the Liouville approach. The reader may find details in ref. [7]. Physically this means that such a formalism is capable of describing energy-loss-free transport in biological cells, thereby providing a realization of Fröhlich's ideas [2].

In this article we focus our attention on the microscopic nature of such coherent states, and try to understand their emergence by as much 'conventional' physics arguments as possible. By 'conventional' we mean scenaria based on quantum electrodynamics, which are the major interactions expected to dominate at the energy scales of the MT 2 . We make an attempt to pick up possible sources of decoherence,

\footnotetext{
${ }^{1}$ It is however, possible, that such a distortion involves a sufficient movement of mass so as to create a virtual microscopic singularity in the surrounding space time [8, 9, 5]; this could then cause collapse of the macroscopic quantum state, leading to conscious perception. Such daring assumptions/conjectures have gain some support by the fact that in certain physical models of MT dynamics [5] the estimated time of collapse is of order $\mathcal{O}(1 \mathrm{sec})$, which is in excellent qualitative agreement with a plethora of experimental/observational findings in Neurobiology. In the present work we shall not discuss these issues.

${ }^{2}$ According to the above discussion, then, quantum gravity entanglement could be, if at all, important for the decoherence of the coherent state formed as a result of the water-dimer coupling.
} 
consistent with the above-mentioned energy-loss-free transfer scenario in biological cells, and estimate the associated time scales.

The structure of this article is a follows: in section 2 we review briefly the physical one-dimensional model describing MT dynamics, and discuss briefly the mathematical background for obtaining solitonic structures. An important rôle for the existence of the solitonic structures is played by the ordered water in the interior of the MT. The importance of the water in the interior of the MT has been emphasized by S. Hameroff, in connection with information processing, already some time ago [15, 16]. In our article we present a scenario for the formation of electric-dipole quantum coherent modes in the water of the MT, inspired by earlier suggestions on 'laser-like' behaviour of the water [17, 18, 19]. Such coherent modes couple with the unpaired electrons of the MT dimers.

In this work we conjecture that such a coupling would result in the so-called Vacuum Field Rabi Splitting (VFRS) [20], a sort of dynamical Stark effect occuring in the emission or absorption spectra of the dimers as a result of the vaccum quantum fluctuations. This phenomenon is characteristic of the behaviour of atoms inside quantum electrodynamical cavities, the rôle of which is played in our work by the MT cylindrical structures themselves.

A review of this phenomenon in the atomic physics is given in section 3 . The atomic Rabi Splitting is a consequence of the coupling of the atoms with the coherent modes of electromagnetic radiation inside the cavity. It is considered by many as a 'proof' of the quantum nature of the electromagnetic radiation [20]. In our work, we consider this phenomenon, if true, as a manifestation of the quantum mechanical nature of the MT arrangement inside the cell.

This is discussed in section 4 , where we present a scenario according to which the MT arrangements inside the cell act as fairly isolated cavities. The ordered-water molecules in their interior, then, provides an environment, necessary to form coherent quantum modes (dipole quanta) [17, 18, 19], whose coupling with the dimers results in VFRS in the respective absorption spectra. Due to dissipation from the cavity walls, there is decoherence of the combined dimer-cavity system coherent state. The resulting decoherence time scales are estimated, for various sources of envirnoments. The main conclusion of our analysis is that in order for the quantum coherent states to play a rôle in dissipationless energy transfer, i.e. the decoherence time to be larger than the the scale required for energy transfer in MT - estimated to be of $\mathcal{O}\left(5 \times 10^{-7} \mathrm{sec}\right)$, for a moderately long MT, in the model of ref. [4] - the damping time scales of the MT cavities must be $T_{r} \geq 10^{-4}-10^{-5} \mathrm{sec}$.

Finally, in section 5, we present our conclusions and suggest some epxeriments to test the above-conjectured quantum mechanical origin of MT arrangements inside the cell. The experiments are inspired by the physics of Rydberg atoms in

However this would require extreme isolation of the MT system, so that any conventional decoherence due to ordinary environmental entanglement is suppressed. Such isolated MT subsystems have been conjectured to occur in certain parts of brain, in which case quantum gravity plays a rôle in consciousness by inducing a collapse of the coherent-state of the MT dimers [5, 8, 9]. At present, we do not know whether this is feasible inside the brain. 
electromagnetic cavities [21, 22].

\section{Review of the Physical Model for Microtubule Dynamics}

\subsection{Classical Considerations}

MicroTubules (MT) are hollow cylinders comprised of an exterior surface (of crosssection diameter $25 \mathrm{~nm}$ ) with 13 arrays (protofilaments) of protein dimers called tubulines. The interior of the cylinder (of cross-section diameter $14 \mathrm{~nm}$ ) contains ordered water molecules, which implies the existence of an electric dipole moment and an electric field. The arrangement of the dimers is such that, if one ignores their size, they resemble triangular lattices on the MT surface. Each dimer consists of two hydrophobic protein pockets, and has an unpaired electron. There are two possible positions of the electron, called $\alpha$ and $\beta$ conformations. When the electron is in the $\beta$-conformation there is a $29^{\circ}$ distortion of the electric dipole moment as compared to the $\alpha$ conformation.

In standard models for the simulation of the MT dynamics, the 'physical' degree of freedom - relevant for the description of the energy transfer - is the projection of the electric dipole moment on the longitudinal symmetry axis (x-axis) of the MT cylinder. The $29^{\circ}$ distortion of the $\beta$-conformation leads to a displacement $u_{n}$ along the $x$-axis, which is thus the relevant physical degree of freedom. This way, the effective system is one-dimensional (spatial), and one has the possibility of a quantum integrable system [5].

Information processing occurs via interactions among the MT protofilament chains. The system may be considered as similar to a model of interacting Ising chains on a trinagular lattice, the latter being defined on the plane stemming from fileting open and flatening the cylindrical surface of MT. Classically, the various dimers can occur in either $\alpha$ or $\beta$ conformations. Each dimer is influenced by the neighboring dimers resulting in the possibility of a transition. This is the basis for classical information processing, which constitutes the picture of a (classical) cellular automatum.

The quantum computer character of the MT network results from the assumption that each dimer finds itself in a superposition of $\alpha$ and $\beta$ conformations [8]. Viewed as a two-state quantum mechanical system, the MT tubulin dimers couple to conformational changes with $10^{-9}-10^{-11}$ sec transitions, corresponding to an angular frequency $\omega \sim \mathcal{O}\left(10^{10}\right)-\mathcal{O}\left(10^{12}\right) \mathrm{Hz}$. In the present work we assume the upper bound of this frequency range to represent (in order of magnitude) the characteristic frequency of the dimers, viewed as a two-state quantum-mechanical system:

$$
\omega_{0} \sim \mathcal{O}\left(10^{12}\right) \mathrm{Hz}
$$

The scenario for quantum computation in MT presuposes that there exists a macroscopic coherent state among the various chains. Let us now try to understand 
its emergence. Let $u_{n}$ be the displacement field of the $n$-th dimer in a MT chain. The continuous approximation proves sufficient for the study of phenomena associated with energy transfer in biological cells, and this implies that one can make the replacement

$$
u_{n} \rightarrow u(x, t)
$$

with $x$ a spatial coordinate along the longitudinal symmetry axis of the MT. There is a time variable $t$ due to fluctuations of the displacements $u(x)$ as a result of the dipole oscillations in the dimers. At this stage, $t$ is viewed as a reversible variable.

The effects of the neighboring dimers (including neighboring chains) can be phenomenologically accounted for by an effective potential $V(u)$. In the model of ref. [4] a double-well potential was used, leading to a classical kink solution for the $u(x, t)$ field. More complicated interactions are allowed in the string picture, as explained in ref. [5]. More generic polynomial potential have also been considered in ref. [5].

The effects of the surrounding water molecules can be summarized by a viscuous force term that damps out the dimer oscillations,

$$
F=-\gamma \partial_{t} u
$$

with $\gamma$ determined phenomenologically at this stage. This friction should be viewed as an environmental effect, which however does not lead to energy dissipation, as a result of the non-trivial solitonic structure of the ground-state and the non-zero constant force due to the electric field. This is a well known result, directly relevant to energy transfer in biological systems [3].

In mathematical terms the effective equation of motion for the relevant field degree of freedom $u(x, t)$ reads:

$$
u^{\prime \prime}(\xi)+\rho u^{\prime}(\xi)=P(u)
$$

where $\xi=x-v t, v$ is the velocity of the soliton, $\rho \propto \gamma$ [ 4 , and $P(u)$ is a polynomial in $u$, of a certain degree, stemming from the variations of the potential $V(u)$ describing interactions among the MT chains [5]. In the mathematical literature [23] there has been a classification of solutions of equations of this form. For certain forms of the potential, the solutions include kink solitons that may be responsible for dissipation-free energy transfer in biological cells f. A typical propagation velocity

\footnotetext{
${ }^{3}$ In the 'string picture of $\mathrm{MT}$ ' the various solutions are related [5 by appropriate Renormalization-Scheme changes on the world-sheet of the string (generalizaing appropriately the particle like structures). This is reflected in certain ambiguities in the form of the string potential $V(u)$; what seems to be a unique phenomenological potential in point-like theory, determining various forms of soliton solutions, may not have such a unique interpretation in the completelyintegrable (non-critical) string picture of MT. This suggests that the point-like field theory soliton solutions, discussed above, constitute only a 'low-energy' approximation of more complicated (solitonic) ground states of the string. As discussed in the context of Liouville strings in ref. [5], such stringy ground states share similar properties with their point-like theory counterparts as far as dissipation-free energy transfer is concerned.
} 
of the kink solitons of ref. [4] is $v \sim 2 \mathrm{~m} / \mathrm{sec}$. This implies that for moderately long microtubules, of length $L \sim 10^{-6} \mathrm{~m}$, such kinks transport energy in

$$
t_{F} \sim 5 \times 10^{-7} \mathrm{sec}
$$

This scale is larger than the time scale that Fröhlich had conjectured as corresponding to the frequency of the coherent phonons in biological matter $\left(t \sim 10^{-11}-10^{-12}\right.$ $s$ ). In this article, however, we shall keep calling the time (5) the Fröhlich scale, since, upon quantization, the kink soliton solutions of ref. [4] yield quantum coherent states [5] that are very similar to Fröhlich's phonons.

Therefore, in the above 'phenomenological' approach to the MT physics, the importance of the water environment can be seen formally as follows: were it not for the friction term (3) there would be no stable solitonic structures in the ferroelectric distortive model of ref. [4] 国.

\subsection{Quantum Considerations}

Let us attempt a microscopic analysis of the physics underlying the interaction of the water molecules with the dimers of the MT. Our investigation points towards the fact that, as a result of the ordered structure of the water environment, there appear collective coherent modes, which in turn interact with the dimer structures (mainly through the unpaired electrons of the dimers) leading to the formation of a quantum coherent solitonic state that extends over the whole network of MT. According to the idea put forward in ref. [5], following ref. [6], such coherent states should be viewed as the result of decoherence of the dimer system due to its interaction/coupling with the water environment.

As we shall argue below, such a coupling could be detected by a phenomenon analogous to what is happening in atoms interacting with coherent modes of the electromagnetic radiation in Cavities, namely the Vacuum-Field Rabi Slitting (VFRS) [20]. Our conjecture is that the interior of MT, full of ordered water molecules, can be viewed as a cavity rather than a wave guide; the cavity structure can be formed by 'closing' the ends of the MT, and is a way of providing a fairly isolated system which can sustain coherent modes.

This type of environmental entanglement is described in the Liouville framework by the world-sheet renormalization-group $\beta$-function, $\beta^{u}$, of the displacement field, $u(x)$, of the dimers, decribed above. A non-trivial $\beta^{u} \neq 0$ expresses deviations from conformal invariance, as a consequence of environmental entanglement [5]. The $\beta$-function corresponds to an energy-dependent environmental coupling, since its magnitude depends on the energy of the field $u(x)$ [5]:

$$
\beta^{u}=\mathcal{O}\left[\left(E / M_{s}\right)^{2}\right]
$$

\footnotetext{
${ }^{4}$ In the (non-critical) Liouville string picture such a coupling is responsible for the appearance of the appropriate 'dilaton'-like terms in the world-sheet action, which are essential for consistency of the Liouville string [24, 25, 5].
} 
where $M_{s}$ is a string scale, characteristic of the problem. The scale $M_{s}$ acts as an ultraviolet cut-off for the energies of the low-energy field theory obtained from the 'string', and its size depends on the details of the subsystem $u(x, t)$ as well as the environment.

The Master Equation for the time evolution of the density matrix $\rho$ of the displacement field $u$ in the Liouville approach reads [7, 5]:

$$
\partial_{t} \rho=i[\rho, H]+i \beta^{u}[\rho, u] M_{s}
$$

where $H$ is the Hamiltonian of the dimer chain. The decoherence time in this approach is estimated as [5]:

$$
t_{\text {decoh } / \text { Liouv }} \sim \frac{1}{\mathcal{N} \beta^{u} M_{s}}
$$

where $\mathcal{N}$ denotes the number of dimers in the MT chain. The time scale (8) is the time over which the solitonic quantum coherent pointer states are formed in the MT networks, as a result of the ordered-water-induced decoherence.

We now remark that, in the approach of viewing the MT as cavities, the main source of dissipation will then be the leakage of photons or other coherent modes from the cavity. Its rate can be assumed small for the MT of the brain, otherwise the incoherent mode will dominate. This dissipation constitutes an ordinary environment, which cannot lead to the formation of pointer states, but instead induces eventual collapse of the solitonic states into completely classical ground states.

Hence, according to the above discussion, there are two stages when decoherence plays an important rôle.

- (i) The first stage concerns the coupling of the system of dimers with the coherent modes formed in the ordered water. This coupling pertains to a specific environment which induces decoherence in the dimer sub-system. producing quantum coherent states ('pointer states') according to refs. [6, 5]. The time scale of this decoherence corresponds to the time scale necessary for the formation of the solitonic coherent states of ref. [5], see eq. (8) above.

- (ii) The second stage refers to the decoherence due to ordinary dissipation through the walls of the (imperfect) MT cavities. Such an ordinary environment does not admit a pointer basis [13], but causes collapse of the quantum coherent state of stage (i) down to a classical ground state.

In this article, we shall estimate the decoherence time scales of both stages (i) and (ii) and compare them with the Fröhlich scale. Moreover, we shall propose specific experimental tests for the detection of the quantum-mechanical behaviour of the MT arrangements inside the cell. An important feature of all these tests is, as we mentioned previously, the Vacuum-Field Rabi Splitting phenomenon, to a brief description of which we now turn for instructive purposes. 


\section{On the Rabi Splitting in Atomic Physics and the Quantum Nature of Electromagnetic Radi- ation}

\subsection{Description of the Rabi splitting phenomenon}

In this section we shall recapitulate briefly the VFRS in atomic physics. This phenomenon has been predicted for the emission spectra of atoms inside electromagnetic cavities [20], in an attempt to understand the quantized nature of the electromagnetic radiation. The basic principle underlying the phenomenon is that, in the presence of an interaction among two oscillators in resonance, the frequency degeneracy is removed by an amount proportional to the strength of the coupling. In the cavity $Q E D$ case of ref. [20], one oscillator consists of a small collection of $N$ atoms, whilst the other is a resonant mode of a high- $Q$ (uality) cavity f Immediately after the suggestion of ref. [20], a similar phenomenon has been predicted for absorption spectra of atoms in cavities [27].

By now, the situation has been verified experimentally on a number of occasions 21]. In such experiments one excites the coupled atom-cavity system by a tuneable field probe. The excitation is then found resonant not at the 'bare' atom or cavity frequencies but at the split frequencies of the 'dressed' atom-field system. The spliting is enhanced for collections of atoms. For instance, as we shall review below, for a system of $N$ atoms, the split is predicted to be [27]:

$$
\text { Rabi splitting }=2 \lambda \sqrt{N} \quad 2 \lambda=\text { Rabi splitting of a single atom }
$$

Despite its theoretical prediction by means of quantum mechanical oscillator systems coupled with a quantized radiation field mode in a cavity, at present there seems to be still a debate on the nature of the phenomenon: (i) the dominant opinion is that the Rabi splitting is a manifestation of the quantum nature of the electromagnetic radiation (cavity field), and is caused as a result of an entanglement between the atom and the cavity coherent modes of radiation. It is a sort of Stark effect, but here it occurs in the absence of an external field [20]. This 'dynamical Stark effect' is responsible for a splitting of the resonant lines of the atoms by an amount proportional to the collective atomic-dipole amplitude. (ii) there is however a dual interpretation [28], which claims that the splitting can be observed in optical cavities as well, and it is simply a result of classical wave mechanics inside the cavity, where the atomic sample behaves as a refractive medium with a complex index, which splits the cavity mode into two components.

Irrespectively of this second classical interpretation, one cannot deny the presence of the phenomenon in entangled atom-quantum-coherent mode systems. This is the point of view we shall be taking in this work, in connection with our picture of viewing MT filled with ordered water as cavities. We shall try to make specific

\footnotetext{
${ }^{5}$ The quantity $Q$ is defined as the ratio of the stored-energy to the energy-loss per period [26], by making the analogy with a damped harmonic oscillator.
} 
experimental predictions that could shed light in the formation of quantum coherent states, and their eventual decoherence. As mentioned above, the latter could be due to the interaction of the dimer unpaired spins (playing the rôle of the atoms in the Rabi experiments) with the ordered-water coherent modes (playing the rôle of the cavity fields). Possible scenaria for the origin of such cavity coherent modes will be described below.

Let us first recapitulate briefly the theoretical basis of the Rabi-splitting phenomenon, which will allow the non-expert reader to assess the situation better. We shall present the phenomenon from a point of view that will help us transcribe it directly to the MT case. Consider an atom of a frequency $\omega_{0}$ in interaction with a single coherent mode of electromagnetic radiation field of frequency $\omega$. The relevant Hamiltonian is:

$$
H=\hbar \omega_{0} \sum_{i} S_{i}^{z}+\hbar \omega a^{\dagger} a+\sum_{i}\left(\hbar \lambda S_{i}^{+} a+H . C .\right)
$$

where $a^{\dagger}, a$ are the creation and annihilation cavity radiation field modes, $S_{i}^{z}, S_{i}^{ \pm}$ are the usual spin- $\frac{1}{2}$ operators, and $\lambda$ is the atom-field coupling. The atom-field system is not an isolated system, since there is dissipation due to the interaction of the system with the surrounding world. An important source of dissipation is the leakage of photons from the cavity at some rate $\kappa$. If the rate of dissipation is not too big, then a quantum coherent state can be formed, which would allow the observation of the vacuum-field Rabi oscillations. The density matrix $\rho$ of the atom-field system obeys a Markov-type master equation for the evolution in time $t$ [27]:

$$
\partial_{t} \rho=-\frac{i}{\hbar}[H, \rho]-\kappa\left(a^{\dagger} a \rho-2 a \rho a^{\dagger}+\rho a^{\dagger} a\right)
$$

The limit $\kappa<<\lambda \sqrt{N}$ guarantees the possibility of the formation of a quantum coherent state, i.e. this limit describes environments that are weakly coupled to the system, and therefore the decoherence times (see below) are very long. In this limit one can concentrate on the off-diagonal elements of the density matrix, and make the following ('secular') approximation for their evolution [27]:

$$
\partial_{t} \rho_{i j}=-\frac{i}{\hbar}\left(E_{i}-E_{j}\right) \rho_{i j}-\Gamma_{i j} \rho_{i j}
$$

where $\Gamma_{i j}$ denotes the damping factor, pertaining to the weak coupling of the atomfield system to the environment. The analysis of ref. [27] pertained to the evaluation of the susceptibility tensor of the system, $\chi_{\alpha \beta}$, which can be calculated by considering its interaction of the system with an external field of frequency $\Omega$. The absorption spectrum is proportional to $\operatorname{Im} \chi(\Omega)$. A standard quantum-mechanical computation yields the result [27]:

$$
\begin{aligned}
& \operatorname{Im} \chi(\Omega)=\cos ^{2} \theta \frac{\Gamma_{-} / \pi}{\Gamma_{-}^{2}+\left\{\Omega-\omega_{0}+\Delta / 2-\frac{1}{2}\left(\Delta^{2}+4 N \lambda^{2}\right)^{1 / 2}\right\}^{2}}+ \\
& \sin ^{2} \theta \frac{\Gamma_{+} / \pi}{\Gamma_{+}^{2}+\left\{\Omega-\omega_{0}+\Delta / 2+\frac{1}{2}\left(\Delta^{2}+4 N \lambda^{2}\right)^{1 / 2}\right\}^{2}}
\end{aligned}
$$


with $\Delta \equiv \omega_{0}-\omega$. In the above expression the damping factors $\Gamma_{ \pm}$represent the damping in the equation of motion for the off-diagonal element of the density matrix $<\Psi_{0}|\rho| \Psi_{ \pm}^{S, C}>$ where $\Psi_{ \pm}$are eigenfucntions of $H$, classified by the eigenvalues of the operators $S^{2}$, and $S^{z}+a^{\dagger} a \equiv C$, in particular [27]: $S=N / 2$, and $C=1-N / 2$.

The expression (13) summarizes the effect of Rabi-vacuum splitting in absorption spectra of atoms: there is a doublet structure (splitting) of the absorption spectrum with peaks at:

$$
\Omega=\omega_{0}-\Delta / 2 \pm \frac{1}{2}\left(\Delta^{2}+4 N \lambda^{2}\right)^{1 / 2}
$$

For resonant cavities the splitting occurs with equal weights

$$
\Omega=\omega_{0} \pm \lambda \sqrt{N}
$$

Notice here the enhancement in the effect for multi-atom systems $N>>1$. This is the 'Vacuum Field Rabi Splitting phenomenon', predicted in emission spectra in ref. [20]. As we have already mentioned, the above derivation pertains to absorption spectra, where the situation is formally much simpler [27]. It is also this latter case that is of interest to us for the purposes of this work.

The quantity $2 \lambda \sqrt{N}$ is called the 'Rabi frequency' [20]. From the emissionspectrum theoretical analysis an estimate of $\lambda$ may be inferrred which involves the matrix element, $\underline{d}$, of atomic electric dipole between the energy states of the two-level atom [20]:

$$
\lambda=\frac{E_{v a c} \underline{d} \cdot \underline{\epsilon}}{\hbar}
$$

where $\underline{\epsilon}$ is the cavity (radiation) mode polarization, and

$$
E_{v a c} \sim\left(\frac{2 \pi \hbar \omega_{c}}{\varepsilon_{0} V}\right)^{1 / 2}
$$

is the r.m.s. vacuum field amplitude at the center of the cavity of volume $V$, and of frequency $\omega_{c}$, with $\varepsilon_{0}$ the dielectric constant of the vacuum [: $\varepsilon_{0} c^{2}=\frac{10^{7}}{4 \pi}$, in M.K.S. units. As mentioned above, there are simple experiments which confirmed this effect 21], involving beams of Rydberg atoms resonantly coupled to superconducting cavities.

The situation which is of interest to us involves atoms that are near resonance with the cavity. In this case $\Delta<<\omega_{0}$, but such that $\lambda^{2} N /|\Delta|^{2}<<1$; in such a case, formula (14) yields two peaks that are characterized by dispersive frequency shifts $\propto \frac{1}{\Delta}:$

$$
\Omega \simeq \omega_{0} \pm \frac{N \lambda^{2}}{|\Delta|}+\mathcal{O}(\Delta)
$$

whilst no energy exchange takes place between atom and cavity mode. This is also the case of interest in experiments using such Rabi couplings to construct

\footnotetext{
${ }^{6}$ For cavities containing other dielectric media, e.g. water in the case of the MT, $\varepsilon_{0}$ should be replaced by the dielectric constant $\varepsilon$ of the medium.
} 
Schrödinger's cats in the laboratory, i.e. macro(meso)scopic combinations 'measuring apparatus + atoms' to verify decoherence experimentally. The first experiment of this sort, which confirms theoretical expectations, is described in ref. [29].

Another important issue, which has been used above, and in ref. [29], is the dephasing of the atom as a result of the above-described atom-field Rabi entanglement. To understand better the situation, let us discuss a more generic case, that of a three-state atom, $f, e, g$, with energies $E_{g}>E_{e}>E_{f}$. Suppose one is interested in the transition $f \rightarrow e$ by absorption, in the presence of atoms in interaction with a cavity mode. Calling $D_{e f}^{+} \equiv|e><f|, D_{e f}^{-} \equiv|f><e|=\left(D_{e f}^{+}\right)^{\dagger}$, we have the effective Hamiltonian for the transition $f \rightarrow e$ [30]:

$$
H_{e f f}^{e f}=\hbar \omega_{e f f} D_{e f}^{+} D_{e f}^{-} \quad ; \quad \omega_{e f f}=\omega_{e f}+\frac{\lambda^{2} n}{\Delta}
$$

where the 'effective' frequency $\omega_{\text {eff }}$ incorporates the dispersive frequency shifts (18) of the Rabi effect, appropriate for near-resonant atom-cavity-field systems; $n$ is the number of cavity photons .

Consider now an experiment to measure, say, the photon number $n$ in the cavity. The relevant probe $P$ can be the above-described three-state atom, in a superposition of $e$ and $f$ states. In this picture the photon number $n$ is an eigenvalue of the cavity signal operator $a_{s}^{\dagger} a_{s}$; the interaction Hamiltonian between atom and cavity then reads [30]:

$$
H_{I}=\frac{\hbar \lambda^{2}}{\Delta} a_{s}^{\dagger} a_{s} D_{e f}^{+} D_{e f}^{-}
$$

The probe observable is the atomic dipole operator:

$$
A_{P}=\frac{1}{2 i}\left(D_{e f}^{+}-D_{e f}^{-}\right)
$$

The Heisenberg evolution of this operator yields:

$$
i \hbar \frac{d}{d t} A_{P}=\left[A_{P}, H_{e f f}^{e f}+H_{I}\right]
$$

from which it is easily seen that in a time interval $t$ the phase of the probe is changing by:

$$
\Delta \phi=\omega_{e f} t+\frac{\lambda^{2} n}{\Delta} t
$$

The case of interest for the experiment of ref. [29] is a two-state atom; the resulting phase shift is then obtained from (23) by setting $\omega_{e f}=0$. In that case, the phase entanglement due to the atom-field Rabi coupling is

$$
\Delta \phi_{R}=\frac{\lambda^{2} n}{\Delta} t
$$

\footnotetext{
${ }^{7}$ Notice that the $\sqrt{n}$ characteristic scaling law for the Rabi splitting (15) is also valid in the case of interaction of a single atom with $n$ cavity oscillator quanta (e.g. a coherent cavity mode).
} 
for a near resonance atom-field system, with a small detuning $\Delta$. Such a situation is encountered precisely in the experiment of ref. [29], which will constitute our prototype for the extension of the above results to the MT case. Before doing so it is instructive to review briefly the experiment of [29], where decoherence of an open mesoscopic system ('Schrödinger's cat') has been demonstrated.

\subsection{Rabi splitting and decoherence: experimenal verifica- tion}

The experiment consists of sending a Rubidium atom with two circular Rydberg states $e$ and $g$, through a microwave cavity storing a small coherent field $|\alpha\rangle$. The coherent cavity mode is mesoscopic in the sense of possessing an average number of photons of order $\mathcal{O}(10)$. The atom-cavity coupling is measured by the Rabi frequency $2 \lambda / 2 \pi=48 \mathrm{kHz}$. The condition for Rabi dispersive shifts (18) is satisfied by having a $\Delta / 2 \pi \in[70,800] k H z$.

The set up of the experiment is as follows: The atom is prepared in the superposition of $e, g$ states, by virtue of a resonant microwave cavity $R_{1}$. Then it crosses the cavity $C$, which is coupled to a reservoir that damps its energy (dissipation) at a characteristic time scale $T_{r}<<1.5 . \mathrm{ms}$. Typical cavities, used for atomic scale experiments such as the above, have dimensions which lie in the $\mathrm{mm}$ range or at most $\mathrm{cm}$ range. In the cavity $C$ a number of photons varying from 0 to 10 is injected by a pulse source. The field in the cavity relaxes to vacuum - thereby causing dissipation through leakeage of photons through the cavity - during a time $T_{r}$, before being regenerated for the next atom. The experiment is at an effective temperature of $T=0.6 \mathrm{~K}$, which is low enough so as to minimize thermal effects. After leaving $C$, the atom passes through a second cavity $R_{2}$, identical to $R_{1}$; one then measures the probability of finding the atom in the state, say, $g$. This would mean decoherence. The decoherence time is measured for various photon numbers; this helps testing the theoretical predictions that decoherence between two 'pointer states' of a quantum superposition occurs at a rate proportional to the square of the distance among the states [31, 32, 6, 11].

Let us understand this latter point better. The coherent oscillator states, characterizing the cavity modes, constitute such a pointer basis: an oscillator in a coherent state is defined by the average number of oscillator quanta $n$ as: $|\alpha>:| \alpha \mid=\sqrt{n}$. Then, consider the measurement of the above-described experiment, according to which there is only a phase entanglement between the cavity and the atom: the combined atom-cavity (meter) system is originally in the state

$$
|\Psi>=| e, \alpha e^{i \phi}>+\mid g, \alpha e^{-i \phi}>
$$

According to (24) the dephasing is atomic-level dependent $\phi \propto \lambda^{2} \operatorname{tn} / \Delta$. Coupling the oscillator to a reservoir, that damps its energy in a characteristic time scale $T_{r}$, produces decoherence. According to the general theory [10, 32, 30, 31] the latter occurs during a time scale inversely proportional to the square of the distance 
between the 'pointer' states $D^{2}$ :

$$
t_{\text {collapse }}=\frac{2 T_{r}}{D^{2}}
$$

This is easy to verify in a prototype toy model, involving only a phase entanglement, i.e. without energy dissipation. To this end, consider the Hamiltonian:

$$
H=\hbar \omega a^{\dagger} a+a^{\dagger} a \Gamma
$$

with $a^{\dagger}, a$ creation and annihilation operators of a quantum oscillator, and $\Gamma$ a phase damping. The 'pointer' states for this problem are characterized by the eigenvalues $n$ of the number operator $a^{\dagger} a$ which commutes with the Hamiltonian $\left[a^{\dagger} a, H\right]=0$, i.e. the pointer basis is $\{\mid n>\}$. The pertinent Markov master equation for the density matrix, $\rho$, reads:

$$
\partial_{t} \rho=\frac{\kappa}{2}\left(2 a^{\dagger} a \rho a^{\dagger} a-\rho a^{\dagger} a a^{\dagger} a-a^{\dagger} a a^{\dagger} a \rho\right)
$$

Writing $\rho(t)=\sum_{m, n} \rho_{m n}(t)|n><m|$ it is straightforward to determine the time dependence of $\rho_{n m}(t)$ from (28)

$$
\rho_{n m}(t)=e^{-\kappa(n-m)^{2} t / 2} \rho_{n m}(0)
$$

which implies that the coherence between two different $(m \neq n)$ pointer (number) states is damped at a typical scale given by (26), where $T_{r}=\frac{1}{\kappa}$, and the distance $D$ is given by $n-m$ in the above example. As mentioned previously, this inverse $D^{2}$-behaviour seems to be a generic feature of open (decohering) systems, including open string models [7, 5].

In the set up of ref. 29 the distance $D$ is given by

$$
D=2 \sqrt{n} \sin \phi \simeq 2 n^{3 / 2} \frac{\lambda^{2} t}{\Delta}
$$

for Rabi couplings $2 \lambda$, such that $\lambda^{2} t n<<\Delta$. For mesoscopic systems $n \sim 10, D>1$, and, hence, decoherence occurs over a much shorter time scale than $T_{r}$; in particular, for $\Delta / 2 \pi \sim 70 \mathrm{kHz}$ the decoherence time is $0.24 T_{r}$ [29]. This concludes the construction of a Schrödinger's cat, and the associated 'measurement process'. Notice that the above construction is made in two stages: first it involves an interaction of the atom with the cavity field, which results in a coherent state of the combined 'atom-meter', and then dissipation is induced by coupling the cavity (measuring apparatus) to the environment, which damps its energy, thereby inducing decoherence in the 'atom+meter' system. The important point to realize is that the more macroscopic the cavity mode is (i.e. the higher the number of oscillator quanta), the shorter the decoherence time is. This is exactly what was to be expected from the general theory [6, 10, 11, 7]. 


\section{Rabi Vacuum Coupling in MT and Energy Trans- fer in the Cell}

\subsection{Microscopic Mechanisms for the formation of Coherent States in MT}

Above we have sketched the experimental construction of a mesoscopic quantum coherent state (a 'Schrödinger's cat' (SC)). The entanglement of the atom with the coherent cavity mode, manifested experimentally by the 'vacuum Rabi splitiing', leads to a quantum-coherent state for the combined atom-cavity system (SC), comprising of the superposition of the states of the two-level Rydberg atom. Dissipation induced by the leakage of photons in the cavity leads to decoherence of the coherent atom-cavity state, in a time scale given by (26). This time scale depends crucially on the nature of the coupled system, and the nature of the 'environment'. It is the point of this session to attempt to discuss a similar situation that conjecturally occurs in the systems of MT. We believe that understanding the formation of Schródinger's cats in MT networks will unravel, if true, the mysteries of the brain as a (quantum) computer, which might also be related to the important issue of 'conscious perception', as advocated in refs. [9, 8, 50.

The first issue concerns the nature of the 'cavity-field modes'. Our point in this section is to argue that the presence of ordered water, which seems to occupy the interior of the microtubules [16], plays an important rôle in producing coherent modes, which resemble those of the ordinary electromagnetic field in superconducting cavities, discussed above.

Let us first review briefly some suggestions about the rôle of the electric dipole moment of water molecules in producing coherent modes after coupling with the electromagnetic radiation field [17]. Such a coupling implies a 'laser-like' behaviour. Although it is not clear to us whether such a behaviour characterizes ordinary water, in fact we believe it does not due to the strong suppression of such couplings in the disordered ordinary water, however it is quite plausible that such a behaviour characterizes the ordered water molecules that exist in the interior of MT [16]. If true, then this electric dipole-quantum radiation coupling will be responsible, according to the analysis of ref. [17], for the appearance of collective quantum coherent modes. The Hamiltonian used in the theoretical model of ref. [17] is:

$$
H_{o w}=\sum_{j=1}^{M}\left[\frac{1}{2 I} L_{j}^{2}+\underline{A} \cdot \underline{d}_{e j}\right]
$$

where $A$ is the quantized electromagnetic field in the radiation gauge [17], $M$ is the number of water molecules, $L_{j}$ is the total angular momentum vector of a single molecule, $I$ is the associated (average) moment of inertia, and $d_{e j}$ is the electric dipole vector of a single molecule, $\left|d_{e j}\right| \sim 2 e \otimes d_{e}$, with $d_{e} \sim 0.2$ Angström. As a result of the dipole-radiation interaction in (31) coherent modes emerge, which in ref. [18 have been interpreted as arising from the quantization of the Goldstone 
modes responsible for the spontaneous breaking of the electric dipole (rotational) symmetry. Such modes are termed 'dipole quanta' in ref. 18.

This kind of mechanism has been applied to microtubules [19], with the conclusion that such coherent modes cause 'super-radiance', i.e. create a specific quantummechanical ordering in the water molecules with characteristic times much shorter than those of thermal interaction. In addition, the optical medium inside the internal hollow core of the microtubule is made transparent by the coherent photons themselves [19]. Such phenomena, if observed, could verify the coherent-mode emission from living matter, thereby verifying Fröhlich's ideas.

In our picture of viewing the MT arrangements as cavities, these coherent modes are the quantum coherent 'oscillator' modes of section 3, represented by annihilation and creation operators $a_{c}, a_{c}^{\dagger}$, which play the rôle of the cavity modes, if the orderedwater interior of the MT is viewed as an isolated cavity 8 . The rôle of the small collection of atoms, described in the atomic physics analogue above, is played in this picture by the protein dimers of the MT chains. The latter constitute a two-state system due to the $\alpha$ and $\beta$ conformations, defined by the position on the unpaired spin in the dimer pockets. The presence of unpaired electrons is crucial to such an analogy. The interaction of the dipole-quanta coherent modes with the protein dimers results in an entanglement which we claim is responsible for the emergence of soliton quantum coherent states, extending over large scale, e.g. the MT or even the entire MT network.

The issue, we are concerned with here, is whether such coherent states are responsible for energy-loss-free transport, as well as for quantum computations due to their eventual collapse, as a result of 'environmental' entanglement of the entire 'MT dimers + ordered water' system. An explicit construction of such solitonic states has been made in the field-theoretic model for MT dynamics of ref. [5], based on classical ferroelectric models for the displacement field $u(x, t)$ discussed in section 2 [4]. The quantum-mechanical picture described here should be viewed as a simplification of the field-theoretic formalism, which, however, is sufficient for qualitative estimates of the induced decoherence.

\subsection{Decoherence and dissipationless energy transfer}

To study quantitatively the effects of decoherence in MT systems we make the plaussible assumption that the environmental entanglement of the 'ordered-water cavity' (OWC), which is responsible for dissipation, is attributed entirely to the leakage

${ }^{8}$ This was not the picture envisaged in ref. [17]. However, S. Hameroff, as early as 1974, had conjectured the rôle of MT as 'dielectric waveguides' for photons [15, and in ref. 19] some detailed mathematical construction of the emergence of coherent modes out of the ordered water are presented. In our work in this article we consider the implications of such coherent modes for the system of dimers, in particular for the formation of kink solitons of ref. 田. Thus, our approach is different from that in refs. [17, 19], where attention has been concentrated only on the properties of the water molecules. We should emphasize that the phenomenon of optical transparency due to super-radiance may co-exist with the formation of kink soliton coherent states along the dimer chains, relevant to the dissipation-free energy transfer along the MT, discussed in the present work. 
of photons (electromagnetic radiation quanta) from the MT interior of volume $V$ ('cavity'). This leakage may occur from the nodes of the MT network, if one assumes fairly isolated interia. This leakage will cause decoherence of the coherent state of the 'MT dimer-OWC' system. The leakage determines the damping time scale $T_{r}$ in $(26)$.

The dimers with their two conformational states $\alpha, \beta$ play the rôle of the collection of $N$ two-level Rydberg atoms in the atomic physics analogue described in section 3. If we now make the assumption that the ordered-water dipole-quantum coherent modes couple to the dimers of the MT chains in a way similar to the one leading to a Rabi splitting, described above, then one may assume a coupling $\lambda_{0}$ of order:

$$
\lambda_{0} \sim \frac{d_{\text {dimer }} E_{o w}}{\hbar}
$$

where $d_{\text {dimer }}$ is the single-dimer electric dipole matrix element, associated wiith the transition from the $\alpha$ to the $\beta$ conformation, and $E_{\text {ow }}$ is a r.m.s. typical value of the amplitude of a coherent dipole-quantum field mode.

Given that each dimer has a mobile charge [1]: $q=18 \times 2 e, e$ the electron charge, one may estimate

$$
d_{\text {dimer }} \sim 36 \times \frac{\varepsilon_{0}}{\varepsilon} \times 1.6 . \times 10^{-19} \times 4.10^{-9} \sim 3 \times 10^{-18} \mathrm{Cb} \times \text { Angstrom }
$$

where we used the fact that a typical distance for the estimate of the electric dipole moment for the 'atomic' transition between the $\alpha, \beta$ conformations is of $\mathcal{O}(4 \mathrm{~nm})$, i.e. of order of the distance between the two hydrophobic dimer pockets. We also took account of the fact that, as a result of the water environment, the electric charge of the dimers appears to be screened by the relative dielectric constant of the water, $\varepsilon / \varepsilon_{0} \sim 80$. We note, however, that the biological environment of the unpaired electric charges in the dimer may lead to further suppression of $d_{\text {dimer }}$ (33).

The amplitude of the collective modes of the dipole-quanta may be estimated using the formula (17). In our case the 'cavity volume' is:

$$
V \sim 5 \times 10^{-22} \mathrm{~m}^{3}
$$

which is a typical MT volume, for a moderately long MT $L \sim 10^{-6} \mathrm{~m}$, considered as an isolated cavity 9 , and $\omega_{c}$ a typical frequency of the dipole quanta collective mode dynamics. To estimate this frequency we assume, following the 'super-radiance' model of ref. [19, that the dominant modes are those with frequencies in the

\footnotetext{
${ }^{9}$ Here we consider only a single unit of the MT network. The MT network consists of a large number of such cavities/MT. Our scenario here is to examine soliton formation in a single (isolated) cavity MT, with the only source of dissipation the leakage of photons. This would explain the formation of kinks in a single MT [4, 5]. If the entire network of MT is viewed as a cavity, then the volume $V$ is complicated, but in that case the volume can be estimated roughly as $\mathcal{N}_{m t} V$, with $V$ the average volume of each MT, and $\mathcal{N}_{m t}$ the number of MT in the network population. In such a case, the solitonic state extends over the entire network of MT. It is difficult to model such a situation by simple one-dimensional Hamiltonians as in refs. [4, 5].
} 
range $\omega_{c} \sim \epsilon / \hbar$, where $\epsilon$ is the energy difference between the two principal energy eigenstates of the water molecule, which are assumed to play the dominant rôle in the interaction with the (quantized) electromagnetic radiation field. For the water molecule:

$$
\hbar \omega_{c} \sim 4 \mathrm{meV}
$$

which yields

$$
\omega_{c} \sim \epsilon / \hbar \sim 6 \times 10^{12} s^{-1}
$$

This is of the same order as the characteristic frequency of the dimers (1), implying that the dominant cavity mode and the dimer system are almost in resonance. Note that this is a feature shared by the Atomic Physics systems in Cavities examined in section 3, and thus we may apply the pertinent formalism to our system.

From (17) one obtains for the r.m.s $E_{\text {ow }}$ in order of magnitude:

$$
E_{\text {ow }} \sim 10^{4} \mathrm{~V} / \mathrm{m}
$$

where we took into account the relative dielectric constant of water $\varepsilon / \varepsilon_{0} \sim 80$.

It has not escaped our attention that the electric fields of such order of magnitude can be provided by the electromagnetic interactions of the MT dimer chains, the latter viewed as giant electric dipoles [4]. This may be seen to suggest that the superradiance coherent modes $\omega_{c}$, which in our scenario interact with the unpaired electric charges of the dimers and produce the kink solitons along the chains, owe their existence to the (quantized) electromagnetic interactions of the dimers themselves.

We assume that the system of $\mathcal{N}$ MT dimers interacts with a single dipolequantum mode of the ordered water and we ignored interactions among the dimer spins 10 . In our work here we concentrate our attention on the formation of a coherent soliton along a single dimer chain, the interactions of the remaining 12 chains in a protofilament MT cylinder being represented by appropriate interaction terms in the effective potential of the chain MT model of ref. [1]. In a moderately long microtubule of length $L \simeq 10^{-6} \mathrm{~m}$ there are

$$
\mathcal{N}=L / 8 \simeq 10^{2}
$$

tubulin dimers of average length $8 \mathrm{~nm}$ in each chain.

Then, from (32), (33), and (37), the conjectured Rabi-vacuum splitting, describing the entanglement of the interior coherent modes with the dimers can be estimated to be of order

$$
\text { Rabi coupling for } \mathrm{MT} \equiv \lambda_{M T}=\sqrt{\mathcal{N}} \lambda_{0} \sim 3 \times 10^{11} s^{-1}
$$

which is, on average, an order of magnitude smaller than the characteristic frequency of the dimers (四). In this way, the perturbative analysis of section 3, for small Rabi

\footnotetext{
${ }^{10}$ More complicated situations, including interactions among the dimers, as well as of the dimers with more than one radiation quanta, which might undoubtedly occur in nature, complicate the above estimate.
} 
splittings $\lambda<<\omega_{0}$, is valid. Indeed, as can be seen from (四),(36), the detuning $\Delta$ is of order:

$$
\Delta / \lambda_{0} \sim \mathcal{O}(10)-\mathcal{O}(100)
$$

implying that the condition $\lambda_{0}^{2} \mathcal{N} /|\Delta|^{2}<<1$, necessary for the perturbative analysis of section 3, is satisfied. The Rabi frequency (39) corresponds to an energy splitting $\hbar \lambda_{M T} \sim 0.1 \mathrm{meV}$ for a moderately long $\mathrm{MT}$.

Having estimated the Rabi coupling between the dimers and the ordered-water coherent modes we now proceed to estimate the average time scale necessary for the formation of the pointer coherent states which arise due to this entanglement [6, 5]. This is the same as the decoherence time due to the water-dimer coupling. In such a case one may use the Liouville model of MT [5], discussed in section 2.2, which is known to possess pointer coherent states [14]. The relevant decoherence time scale is given by (8).

For an accurate estimate of the decoherence time one should have a precise knowledge of the violations of conformal invariance associated with the water-dimer coupling. Unlike the quantum-gravity induced decoherence case, where a microscopic model for the description of the distortions caused in the environment of the chain by the external stimuli is available [5], at present a precise conformal-fieldtheory model for the description of the water-dimer coupling is lacking.

However, for our purposes here, it suffices to use our generic approach to the string-theory representation of MT chains, described in ref. [0], where a good orderof-magnitude estimate for $\beta^{u}$ is provided by (6). We, therefore, merely need to have an estimate of the scales $E$ and $M_{s}$ for the problem at hand.

To estimate the 'string' scale $M_{s}$ in our case $\rrbracket$, where only quantum-electromagnetic interactions enter, we notice that in the standard string theory $M_{s}$ acts as an ultraviolet cut-off in the energy of the effective target-sace field theory, in our case the field theory of the displacement field $u(x, t)$. Since in the ferroelectric chain the closest distance separating two unpaired electrons, which are assumed to have the dominant interactions with the water, is the size of a tubulin dimer $\sim d_{\min }=4 \mathrm{~nm}$, an order of magnitiude estimate of $M_{s}$ is:

$$
M_{s} \sim \hbar v_{0} / d_{\min } \sim 1.5 \times 10^{-4} \mathrm{eV},
$$

where we took into account that the Liouville model for MT in ref. [5] is formally a relativistic string model, but with the rôle of the velocity of light played by the sound velocity $v_{0}=1 \mathrm{Km} / \mathrm{sec}$ in organic biological materials. It is interesting to note that this cut-off in energies is less than the kinetic energies of fast kinks, propagating with the valocity of sound. Indeed such energies are of order $E_{0} \sim 10^{-2} \mathrm{eV}$. Such fast kinks have been associated in ref. [5] with quantum gravitational effects, due to

\footnotetext{
${ }^{11}$ In ref. [5], where scenaria for gravity-induced conscious perception were discussed, the scale $M_{s}$ was taken to be the Planck scale $10^{19} \mathrm{GeV}$. In that case decoherence was induced by the coupling of the entire network of brain MT to quantum-gravity fluctuations due to the distortion of the surrounding space time, as result of abrupt conformational changes in the dimers, caused by external stimuli $9,8,5$.
} 
abrupt distortions of space time, and hence their exclusion in the approach of the present work, by considering kinks with energies much less than those, as implied by $M_{s}$, is consistent with our considering only electromagnetic interactions among the dimers and its environment.

The above estimate allows us to get an idea of the typical energy scales of the excitations of the MT systems that dominate the ordered-water-dimer coupling, in the above scenario, and lead to decoherence. As discussed in ref. [5], the dominant part of the energy of a kink in the model of ref. [4] is of order $1 \mathrm{eV}$, and thus much higher than the $M_{s}$ (41). Such scales may play a rôle in the decoherence due to quantum-gravity entanglements [5], which however is much weaker than the electromagnetic ones considered here. On the other hand, as we shall argue now, a typical energy scale for the dimer displacement field $u(x, t)$, much smaller than $M_{s}$, and therefore consistent with our low-energy approach, is provided by the kinetic energy of the kink, which is estimated to be of order [4]

$$
E_{k i n} \simeq 5 \times 10^{-8} \mathrm{eV}
$$

As we shall argue below, this energy scale may be used for our estimate of the time scale (8), which is necessary for the formation of the coherent state due to the waterdimer coupling. We stresss that (42) should not be considered as a typical energy scale associated with the excitation spectrum of the dimers, pertaining to the Rabi splitting discussed above. It is rather an 'effective scale', characterising 'friction' effects between the dimers and the water environment. The latter are responsible [5] for decoherence and the eventual formation of the kink coherent states, which are minimum-entropy states [6], least susceptible to the effects of the water environent.

To justify the above estimate, one should notice that the formation of coherent quantum states through decoherence due to friction [6] is the quantum analogue of the 'drift velocity' acquired by a Brownian particle in classical mechanics. In the (non-relativistic) conformal-field-theory setting of ref. [5] such a friction could be described by the formation of point-like defects on the dimer chains, which could be described by appropriate non-relativistic membrane backgrounds in the $1+1$ dimensional string theory representation of the MT dynamics. Such membranes are stringy defects, which, for instance, could describe the result of an abrupt conformational change of a given dimer due to its coupling with the water environment. Scattering of stringy excitations $u(x, t)$ off the defect causes 'recoil' of the latter, which starts moving with a velocity $v_{d}$. The recoil is due to quantum fluctuations as argued in ref. [33. Such recoil effects are necessary for energy and momentum conservation in the case of (non-relativistic) heavy membranes, considered in ref. [33]. That model represents a pilot model for discussing decoherence due to water environment in our case.

The important feature to notice is that the 'effective mass' of the defect in such a stringy representation turns out to be inverssely proprotional to the string-coupling constant $g_{s}$ [33],

$$
m_{\text {defect }}=\left(8 \sqrt{2} \pi g_{s}\right)^{-1}
$$


which, in turn, depends on the vacuum expectation value of the effective 'dilaton' field $\Phi: g_{s}=e^{-\langle\Phi\rangle}$. In the case at hand, as discussed in ref. [5], the dilaton field is proprotional to the friction coefficient $\gamma$, so that $g_{s} \sim \exp (-\gamma)$ in order of magnitude. Strong friction, therefore, between water molecules and dimers, which may be assumed in our physical model of MT, implies weak string coupling, and hence the non-relativistic approximation for the membranes proves sufficient.

Within the framework of identifying target time with the Liouville mode [7], then, the analysis of [33] has shown that the quantum recoil degrees of freedom of the membrane defect carry information and induce decoherence of the dimer (string) subsystem, corresponding to violations of conformal invariance of order:

$$
\beta^{\text {recoil }} \sim \mathcal{O}\left(v_{d}^{2} / 16 \pi g_{s} M_{s}\right)
$$

where $v_{d}^{2}$ is the recoil velocity of the defect. By energy-momentum conservation, the kinetic energy of the recoiling (non-relativistic) defect is at most of the same order as the kinetic energy of the displacement field (42). This would yield a lower bound on the decoherence time (8) estimated on the basis of (44) to be of order:

$$
t_{\text {owdecoh }} \gtrsim 10^{-10} \mathrm{sec}
$$

for a moderately long MT, with $\mathcal{N}=10^{2}$ dimers. This is the time scale over which solitonic coherent pointer states in the MT dimer system are formed ('pumped'), according to our scenario. Note that the scale (45) is not far from the original Fröhlich scale [2], $10^{-11}-10^{-12}$ sec.

To answer the question whether quantum coherent pointer states are responsible for loss-free energy transport across the MT one should examine the time scale of the decoherence induced by the coupling of the MT to their biological environment as a consequence of dissipation through the walls of the MT cylinders. Such an ordinary environmental entanglement has been ignored in the derivation of (45). It is this environment that will induce decoherence and eventual collapse of the pointer states formed by the interaction of the dimers with the coherent modes in the ordered water. Using typical numbers of MT networks, we can estimate this decoherence time in a way similar to the corresponding situation in atomic physics (26, 30):

$$
t_{\text {collapse }}=\frac{T_{r}}{2 n \mathcal{N} \sin ^{2}\left(\frac{\mathcal{N} n \lambda_{0}^{2} t}{\Delta}\right)}
$$

where we took into account that the dominant (dimer)-(dipole quanta) coupling occurs for ordered-water 'cavity' modes which are almost at resonance with the dimer oscillators (c.f. (36),(10), slightly detuned by $\Delta: \lambda_{0} / \Delta<<1$, c.f. (40).

We also assume that a typical coherent mode of dipole/quanta contains an average of $n=\mathcal{O}(1)-\mathcal{O}(10)$ oscillator quanta. The macroscopic character of the Schrödinger's cat dimer-dipole-quanta system comes from the N dimers in a MT (or $\mathcal{N}_{m t} \mathcal{N}$ in MT networks) [5].

The time $t$ appearing in (46) represents the 'time' of interaction of the dimer system with the dipole quanta. A reasonable estimate of this time scale in our MT 
case can be obtained by equating it with the average life-time of a coherent dipolequantum state, which, in the super-radiance model of ref. [19 can be estimated as

$$
t \sim \frac{c \hbar^{2} V}{4 \pi d_{e j}^{2} \epsilon N_{w} L}
$$

with $d_{e j}$ the electric dipole moment of a water molecule, $L$ the length of the MT, and $N_{w}$ the number of water molecules in the volume $V$ of the MT. For typical values of the parameters for moderately long MT, $L \sim 10^{-6} \mathrm{~m}, N_{w} \sim 10^{8}$, a typical value of $t$ is:

$$
t \sim 10^{-4} \mathrm{sec}
$$

We remark at this point that this is considerably larger than the average life-time of a coherent dipole quantum state in the water model of ref. [17]. Indeed in that model, the corresponding life-time is estimated to be:

$$
t \sim 2 \pi / \omega_{0}
$$

where $\omega_{0} \sim 1 / I$, is a typical frequency of resonating electromagnetic mode in the ordered water. For a typical value of the water molecule moment of intertia [17] this yields a time-scale associated with the coherent interaction $\mathcal{O}\left(10^{-14} \mathrm{sec}\right)$.

The time scale $T_{r}$, over which a cavity MT dissipates its energy, can be identified in our model with the average life-time (47) of a coherent-dipole quantum state:

$$
T_{r} \sim t \sim 10^{-4} \mathrm{sec}
$$

which leads to a naive estimate of the quality factor for the MT cavities, $Q_{M T} \sim$ $\omega_{c} T_{r} \sim \mathcal{O}\left(10^{8}\right)$. We note, for comparison, that high-quality cavities encountered in Rydberg atom experiments dissipate energy in time scales of $\mathcal{O}\left(10^{-3}\right)-\mathcal{O}\left(10^{-4}\right)$ sec, and have $Q$ 's which are comparable to $Q_{M T}$ above. Thus, it is not unreasonable to expect that conditions like (50), characterizing MT cavities, are met in Nature.

From (46), (39), and (50), one then obtains the following estimate for the collapse time of the kink coherent state of the MT dimers due to dissipation:

$$
t_{\text {collapse }} \sim \mathcal{O}\left(10^{-7}\right)-\mathcal{O}\left(10^{-6}\right) \text { sec }
$$

which is larger or equal than the Fröhlich scale (5) required for energy transport across the MT by an average kink soliton in the model of ref. [4]. The result (51), then, implies that Quantum Physics may be responsible for dissipationless energy transfer across the MT.

We close this section by remarking that if the condition $T_{\text {collapse }} \gtrsim 5 \times 10^{-7}$ sec is not met, then the above picture, based on mesoscopic coherent states, would be inconsistent with energy-loss free energy transport, since decoherence due to environmental entanglement would occur before energy could be transported across the MT by the preformed quantum soliton. In such a case energy would be transported due to different mechanisms, one of which is the classical solitons scenario of ref. [由, 3]. However, even in such cases of fast decoherence, the Rabi coupling predicted above could still exist and be subjected to experimental verification. 


\section{Outlook: Possible Experimental Tests Using Micro- Tubules as Cavities}

In the present work we have put forward a conjecture concerning the representation of the MT arrangements inside the cell as isolated high-Q(uality) cavities. We presented a scenario according to which the presence of the ordered water in the interior of the cylindrical arrangements results in the appearance of electric dipole quantum coherent modes, which couple to the unpaired electrons of the MT dimers via Rabi vacuum field couplings, familiar from the physics of Rydberg atoms in electromagnetic cavities [20]. In quantum optics, such couplings are considered as experimental proof of the quantized nature of the electromagnetic radiation. In our case, therefore, if present, such couplings could indicate the existence of the coherent quantum modes of electric dipole quanta in the ordered water environment of MT, conjectured in ref. [18, 17], and used in the present work.

Some generic decoherence time estimates, due to environmental entanglement of the MT cavities, have been given. The conclusion was that only in fairly isolated cavities, which we conjecture exist inside the biological cells, decoherence occurs in time scales which are in agreement with the Fröhlich scale $\left(\sim 5 \times 10^{-7} \mathrm{sec}\right)$ for energy transfer across the MT via the formation of kink solitonic structures. In such a case, dissipationless energy transfer might occur in biological systems, an in particular in MT which are the substratum of cells, in much the same way as frictionless electric current transport occurs in superconductors, i.e. via quantum coherent modes that extend over relatively large spatial regions. A phenomenological analysis indicated that for moderately long MT networks such a situation could be met if the MT cavities dissipate energy in time scales of order $T_{r} \sim 10^{-4}-10^{-5}$ sec. This lower bound is comparable to the corresponding scales of atomic cavities [21], $\sim 10^{-4} \mathrm{sec}$, which, in turn, implies that the above scenaria, on dissipationless energy transport as a result of the formation of quantum coherent states, have a good chance of being realized at the scales of MT, which are comparable to those in Atomic Physics.

We have conjectured in this work that an indirect verification of such a mechanism would be the experimental detection of the aforementioned Vacuum field Rabi coupling, $\lambda_{M T}$, between the MT dimers and the ordered water quantum coherent modes. This coupling, if present, could be tested experimentally by the same methods used to measure VFRS in atomic physics [21], i.e. by using the MT themsleves as cavity environments, and considering tuneable probes to excite the coupled dimerwater system. Such probes could be pulses of (monochromatic ) light, for example, passing through the hollow cylinders of the MT. This would be the analogue of an external field in the atomic experiments described above, which would then resonate, not at the bare frequencies of the coherent dipole quanta or dimers, but at the Rabi splitted ones, and this would have been exhibited by a double pick in the absorption spectra of the dimers 21]. By using MT of different sizes one could thus check on

the characteristic $\sqrt{N}$-enhancement of the Rabi coupling for MT systems with $N$ dimers. 
The technical complications that might arise in such experiments are associated with the absence of completely resonant cavities in practice. In fact, from our discussion in this article, one should expect a slight detuning $\Delta$ between the cavity mode and the dimer, of frequency $\omega_{0}$. As discussed in section 3, the detuning produces a split of the vacuum-Rabi doublet into a cavity line $\omega_{0}+\lambda^{2} / \Delta$ and an 'atomic line' $\omega_{0}-\lambda^{2} / \Delta$. In atomic physics there are well established experiments [21, 22 to detect such splittings. In fact, detection of such lines is considered as a very efficient way of 'quantum non-demolition' measurement [30] for small microwave photon numbers. Such atomic physics experiments, therefore, may be used as a guide in performing the corresponding biological experiments involving MT (as cavities), as suggested in the present work.

We believe that such experimental set-ups will help in sheding some light on the issue of the quantum mechanical nature of the MT arrangements, and even on the processes of the transmission of electric signals (stimuli) by the neuronic systems. Clearly much more work needs to be done before even tentative conclusions are reached, concerning the nature of the MT arrangements. However, we believe that the present work constitutes a useful addition to the programme of understanding the nature of the MT arrangements inside the cell, and the associated processes of energy transfer across the cells.

\section{Acknowledgements}

The work of D.V.N. is supported in part by D.O.E. Grant DEFG05-91-GR-40633.

\section{References}

[1] P. Dustin, MicroTubules (Springer, Berlin 1984);

Y. Engleborghs, Nanobiology 1 (1992), 97.

[2] H. Fröhlich, Bioelectrochemistry, ed. by F. Guttman and H. Keyzer (Plenum, New York 1986).

[3] P. Lal, Physics Letters 111A (1985), 389.

[4] M.V. Satarić, J.A. Tuszyński, R.B. Zakula, Phys. Rev. E48 (1993), 589;

this model of MT dynamics is based on the ferroelectric-ferrodistortive model of : M.A. Collins, A. Blumen, J.F. Currie, and J. Ross, Phys. Rev. B19 (1978), 3630..

[5] N.E. Mavromatos and D.V. Nanopoulos, Int. J. Mod. Phys. B11 (1997), 851.

[6] For a comprehensive review see: W.H. Zurek, Phys. Today 44, No. 10 (1991), 36; see also: W.H. Zurek, Phys. Rev. D24 (1981), 1515; 
A.O. Caldeira and A. J. Leggett, Physica (Amsterdam) 121A (1983), 587; Ann. Phys. 149 (1983), 374.

[7] J. Ellis, N.E. Mavromatos and D.V. Nanopoulos, Phys. Lett. B293 (1992), 37; lectures presented at the Erice Summer School, 31st Course: From Supersymmetry to the Origin of Space-Time, Ettore Majorana Centre, Erice, July 4-12 1993 ; hep-th/9403133, Vol. 31 (1994), p.1 (World Sci. );

For a pedagogical review of this approach see: D.V. Nanopoulos, Riv. Nuov. Cim. Vol. 17, No. 10 (1994), 1.

[8] S. Hameroff and R. Penrose, Orchestrated Reduction of Quantum Coherence in Brain Microtubules: a Model of Consciousness, in Towards a science of Consciousness, The First Tucson Discussions and Debates, eds. S. Hameroff et al. (MIT Press, Cambridge MA 1996), p. 507-540.

[9] R. Penrose, The Emperor's New Mind (Oxford Univ. Press 1989); Shadows of the Mind (Oxford Univ. Press 1994);

D.V. Nanopoulos, Theory of Brain Function, Quantum Mechanincs and Superstrings, hep-ph/9505374, Proc. "XV Brazilian National Meeting on Particles and Fields", eds. M.S. Alves et al., Brasilean Phys. Society (1995), p. 28.

[10] J. Ellis, J.S. Hagelin, D.V. Nanopoulos and M. Srednicki, Nucl. Phys. B241 (1984), 381.

[11] J. Ellis, S. Mohanty and D.V. Nanopoulos, Phys. Lett. B221 (1989), 113.

[12] N. Gisin and I. Percival, J. Phys. A26 (1993), 2233.

[13] A. Albrecht, Phys. Rev. D46 (1992), 5504.

[14] J. Ellis, N.E. Mavromatos and D.V. Nanopoulos, Proc. 1st International Workshop on Phenomenology of Unification from Present to Future, 23-26 March 1994, Roma (eds. G. Diambrini-Palazzi et al., World Sci., Singapore 1994), p.187.

[15] S.R. Hameroff, Am. J. Clin. Med. 2 (1974), 163.

[16] S. Hameroff, Ultimate Computing (Elsevier North-Holland, Amsterdam 1987);

S. Hameroff, S. A. Smith, R.C. Watt, Ann. N.Y. Acad. Sci. 466 (1986), 949.

[17] E. Del Giudice, G. Preparata and G. Vitiello, Phys. Rev. Lett. 61 (1988), 1085.

[18] E. Del Giudice, S. Doglia, M. Milani and G. Vitiello, Nucl. Phys. B251 (FS 13) (1985), 375; ibid B275 (FS 17) (1986), 185.

[19] M. Jibu, S. Hagan, S. Hameroff, K. Pribram and K. Yasue, Biosystems 32 (1994), 195. 
[20] J.J. Sanchez-Mondragon, N.B. Narozhny and J.H. Eberly, Phys. Rev. Lett. 51 (1983), 550.

[21] F. Bernardot et al., Europhysics Lett. 17 (1992), 34.

[22] M. Brune et al., Phys. Rev. Lett. 65 (1990), 976.

[23] M. Otwinowski, R. Paul and W.G. Laidlaw, Phys. Lett. A128 (1988), 483.

[24] F. David, Mod. Phys. Lett. A3 (1988), 1651;

J. Distler and H. Kawai, Nucl. Phys. B321 (1989), 509.

[25] I. Antoniadis, C. Bachas, J. Ellis and D.V. Nanopoulos, Phys. Lett. B211 (1988), 393; Nucl. Phys. B328 (1989), 117; Phys. Lett. B257 (1991), 278;

See also D.V. Nanopoulos, in Proc. International School of Astroparticle Physics, HARC (Houston) (World Scientific, Singapore, 1991), p. 183.

[26] S. Haroche and J.M. Raimond, Cavity Quantum Electrodynamics, ed. P. Berman (Academic Press, New York 1994), p.123, and references therein.

[27] G.S. Agarwal, Phys. Rev. Lett. 53 (1984), 1732.

[28] Yifu Zhu et al., Phys. Rev. Lett. 64 (1990), 2499.

[29] M. Brune et al., Phys. Rev. Lett. 77 (1996), 4887.

[30] M. Brune et al., Phys. Rev. A45 (1991), 5193.

[31] Y.R. Shen, Phys. Rev. 155 (1967), 921;

A.S. Davydov and A.A. Serikov, Phys. Stat. Sol. B51 (1972), 57;

B. Ya. Zel'dovich, A.M. Perelomov and V.S. Popov, Sov. Phys. JETP 28 (1969), 308 ;

For a comprehensive review see: V. Gorini et al., Rep. Math. Phys. Vol. 13 (1978), 149.

[32] D.F. Walls and G.J. Milburn, Phys. Rev. A31 (1985), 2403.

[33] J. Ellis, N.E. Mavromatos and D.V. Nanopoulos, hep-th/9609238; and hepth/9704169, Mod. Phys. Lett. A, in press. 